Vietnam Journal of Mechanics, VAST, Vol.42, No. 4 (2020), pp. 385-400

DOI: https://doi.org/10.15625/0866-7136/14897

\title{
OPTIMAL PARAMETERS OF DYNAMIC VIBRATION ABSORBER FOR LINEAR DAMPED ROTARY SYSTEMS SUBJECTED TO HARMONIC EXCITATION
}

\author{
Vu Duc Phuc ${ }^{1, *}$, Tong Van Canh ${ }^{2}$, Pham Van Lieu ${ }^{3}$ \\ ${ }^{1}$ Hung Yen University of Technology and Education, Vietnam \\ ${ }^{2}$ Korea Institute of Machinery and Materials, South Korea \\ ${ }^{3}$ Hanoi University of Industry, Vietnam \\ *E-mail: ketquancs@gmail.com
}

Received: 18 March 2020 / Published online: 16 July 2020

\begin{abstract}
Dynamic vibration absorber (DVA) is a simple and effective device for vibration absorption used in many practical applications. Determination of suitable parameters for DVA is of significant importance to achieve high vibration reduction effectiveness. This paper presents a method to find the optimal parameters of a DVA attached to a linear damped rotary system excited by harmonic torque. To this end, a closed-form formula for the optimum tuning parameter is derived using the fixed-point theory based on an assumption that the damped rotary systems are lightly or moderately damped. The optimal damping ratio of DVA is found by solving a set of non-linear equations established by the Chebyshev's min-max criterion. The performance of the proposed optimal DVA is compared with that obtained by existing optimal solution in literature. It is shown that the proposed optimal parameters allow to obtain superior vibration suppression compared to existing optimal formula. Extended simulations are carried out to examine the performance of the optimally designed DVA and the sensitivity of the optimum parameters. The simulation results show that the improvement of the vibration performance on damped rotary system can be as much as $90 \%$ by using DVA.
\end{abstract}

Keywords: dynamic vibration absorber, torsional excitation, optimazed parameters, rotary systems.

\section{INTRODUCTION}

Vibration control is essential in many engineering fields. Among vibration control methods, the dynamic vibration absorbers (DVAs) are widely applied because of its efficiency, reliability, and rather low expense [1]. The early study on DVA was conducted by Frahm [2]. Ormondroyd and Den Hartog [3] first introduced the concept of the DVA with spring and viscous damper arranged in parallel. Den Hartog proposed in his book the fixed-points theory which helps find out the closed-form optimal parameters of DVA 
attached to undamped structures [4]. That approach mainly aims at reducing the maximum amplitude magnification factor of the primary system, which is still widely used nowadays [5-7]. Since then, a number of optimization criteria have been proposed for optimal design of DVA, in which the $H_{\infty}$ and $H_{2}$ optimizations were employed by many authors [1,8,9]. Nishihara and Asami [10] proposed an analytical solution for the optimal parameters of DVA using $H_{\infty}$ optimization, which minimized the maximum displacement of the primary mass. Shen et al. [11] studied the optimal design of DVA with negative stiffness based on the $H_{\infty}$ optimization. The $H_{2}$ optimization was used to minimize the mean square displacement of the main mass [12], and the power dissipated by the absorber [13]. Yamaguchi [14] found the optimal parameters of DVA using a stability maximization criterion for minimizing the transient vibration of the system. Argentini et al. [15] proposed a closed-form optimal tuning of TMD coupled with an undamped single DOF system forced by a rotating unbalance. Bisegna and Caruso [16] took the exponential time-decay rate of the system transient response as an optimality condition. Then, the closed-form expressions of the optimal exponential time-decay rate were proposed for undamped systems. The other optimization approaches, such as the frequency locus method [17], the min-max criteria [18], and the numerical optimization scheme [19-21], and averaging technique [22] were also proposed.

The above-mentioned studies have provided a comprehensive background to the design optimization of DVAs. However, there have been few studies on DVA for rotary systems with torsional vibration. The torsional vibrations usually result in significant harmful effects on rotating systems. For example, torsional vibration causes the fluctuation in rotational speed of electric motor leading a severe perturbation of the electro-magnetic flux and thus additional oscillation of the electric currents in the motor [13]. Torsional vibration is one of the greatest danger factors for the shaft line and the crankshaft of the marine power transmission system [23]. Minimization of torsional vibration helps to increase the fatigue durability and the efficient functioning of a large turbo-generator [24]. Recently, several attempts have been made to find out the closed-form optimal parameters of DVA used to reduce torsional vibration of undamped rotary system [25]. For the damped rotary system, Phuc et al. [26] have focused on approximating the damped rotary system by an equivalent undamped rotary system by using the least square criterion [27], from which the optimization problem was solved by using the fixed-points theory.

In this paper, the optimal parameters for a DVA attached on damped rotary system under torsional excitation are determined. Approximation approach for lightly damped systems is used to derive approximated solution for the optimum tuning parameter of DVA. Then, the Chebyshev equioscillation theorem is used to find out the optimal damping ratio. This paper is organized as follows. In Section 2, the model of damped rotary system coupled with a DVA is introduced and the system equations of motion are presented. In Section 3, the optimization problems are solved for the optimal parameters of DVA. Section 4 presents the numerical results. The present method is compared to an existing method. Extended numerical results are also presented to examine the performance of the optimal DVA and investigate the effect of mass and damping ratio of damped rotary system on the optimal results. Finally, Section 5 concludes the paper. 


\section{MODEL OF DAMPED ROTARY SYSTEM ATTACHED WITH A DYNAMIC VIBRATION ABSORBER UNDER TORSIONAL EXCITATION}

\subsection{Vibration equations of damped rotary system with dynamic vibration absorber}

Fig. 1 shows the model of the damped rotary system attached DVA where the damped rotary system is the one DOF shaft which has the mass $\left(m_{s}\right)$, inertial momentum $\left(J_{s}\right)$, torsional stiffness $\left(k_{s}\right)$ and internal coefficient of torsional viscous damper $\left(c_{s}\right)$. The DVA consists of a passive disk having inertial momentum $\left(J_{a}\right)$ is connected to the damped rotary system by springs and dampers with stiffness $\left(k_{j}\right)$ and coefficient of viscous damper $\left(c_{j}\right)$, respectively. The springs and dampers are arranged in parallel and they are distributed on the circles with radius $e_{1}$ and $e_{2}$, respectively. The rotary system is damped because the damper $\left(c_{S}\right)$ is imposed in the model.

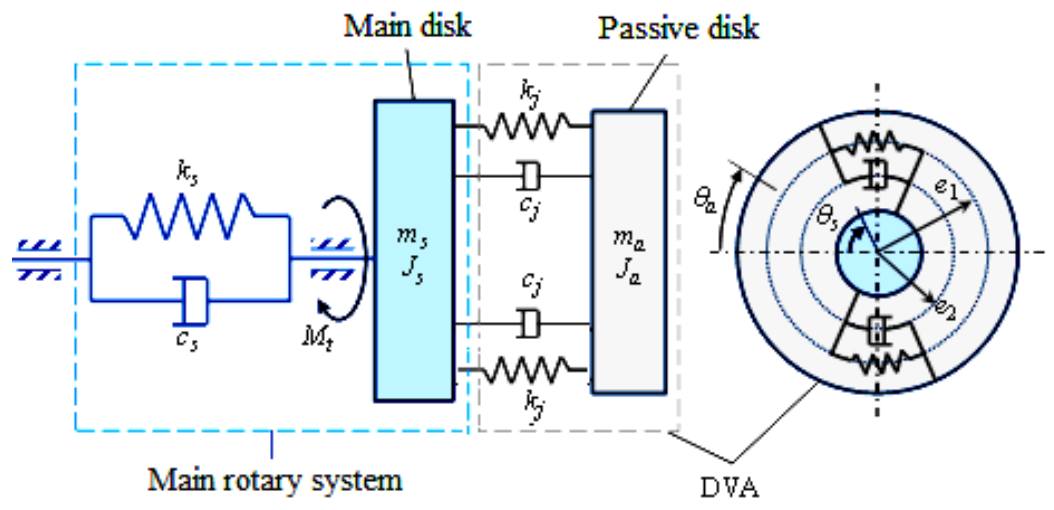

Fig. 1. Model of damped rotary system attached DVA

By applying the Lagrange formulation the differential equations of motion for the system shown in Fig. 1 can be obtained as

$$
\left\{\begin{array}{l}
\left(J_{s}+J_{a}\right) \ddot{\theta}_{s}+J_{a} \ddot{\theta}_{a}+c_{s} \dot{\theta}_{s}+k_{s} \theta_{s}=M_{t}, \\
J_{a}\left(\ddot{\theta}_{r}+\ddot{\theta}_{a}\right)+\sum_{j=1}^{n} c_{j} e_{2}^{2} \dot{\theta}_{a}+\sum_{j=1}^{n} k_{j} e_{1}^{2} \theta_{a}=0 .
\end{array}\right.
$$

In Eqs. (1), $\theta_{s}$ and $\theta_{a}$ are the relative torsional angles of main disk and passive disk, respectively. $M_{t}$ is the harmonic torsional moment given by

$$
M_{t}=M_{0} \sin \Omega t,
$$

where $\Omega$ is the excitation frequency, $e_{1}$ and $e_{2}$ indicate the radial positions of springs and dampers, respectively.

\subsection{Amplitude magnification factor (AMF)}

By describing the harmonic excitation torque in a complex form as

$$
M_{t}=M_{0} e^{i \Omega t}
$$


the solution for Eqs. (1) can be determined as follows

$$
\left\{\begin{array}{l}
\theta_{s}(t)=\hat{\theta}_{s}(\Omega) e^{i \Omega t} \\
\theta_{a}(t)=\hat{\theta}_{a}(\Omega) e^{i \Omega t}
\end{array}\right.
$$

Substituting Eqs. (4) into Eqs. (1), and solving the obtained equation, the relative torsional angle of main disk $\hat{\theta}_{s}$ can be obtained as

$$
\hat{\theta}_{s}=\frac{M_{0}}{k_{s}} \Re\left(\alpha_{j}, \zeta_{j}\right),
$$

where $\Re\left(\alpha_{j}, \zeta_{j}\right)$ is the transfer function of the system described by

$$
\Re\left(\alpha_{j}, \zeta_{j}\right)=\frac{1}{1-\left(1+\mu \eta^{2}\right) \beta^{2}+2 \zeta_{s} \beta i-\frac{\mu^{2} \eta^{4} \beta^{4}}{-\mu \eta^{2} \beta^{2}+\sum_{j=1}^{n}\left(\gamma^{2} \mu \alpha_{j}^{2}+2 \lambda^{2} \mu \beta \alpha_{j} \zeta_{j} i\right)}} .
$$

The other parameters in Eq. (6) are given as follows

$$
\begin{aligned}
& \mu=\frac{m_{a}}{m_{s}}, \quad \eta=\frac{\rho_{a}}{\rho_{s}}, \quad \gamma=\frac{e_{1}}{\rho_{s}}, \quad \lambda=\frac{e_{2}}{\rho_{s}}, \quad \omega_{j}=\sqrt{\frac{k_{j}}{m_{a}}}, \quad \alpha_{j}=\frac{\omega_{j}}{\omega_{s}}, \\
& \zeta_{j}=\frac{c_{j}}{2 m_{a} \omega_{j}}, \quad \omega_{s}=\sqrt{\frac{k_{s}}{J_{s}}}, \quad \zeta_{s}=\frac{c_{s}}{2 J_{s} \omega_{s}}, \quad \beta=\frac{\Omega}{\omega_{s}},
\end{aligned}
$$

where $\mu$ is the mass ratio; $\gamma, \lambda$ and $\eta$ respectively represent the ratio between radial position of springs, radial position of dampers and the gyration radius of passive disk to the gyration radius of main disk; $\omega_{s}$ indicates the natural frequency of rotary system; $\alpha$ and $\zeta$ are the tuning and damping ratios, respectively; $\beta$ is the frequency ratio; the indexes $j$ and $s$ stand for the DVA and main disk, respectively. The amplitude magnification factor $(\mathrm{AMF})$ is defined as the magnitude of the complex transfer function as

$$
H=|\Re(\alpha, \zeta)|=\sqrt{\frac{A \zeta^{2}+B}{C \zeta^{2}+D \zeta+E}} .
$$

In Eq. (8), all the springs and dampers of the DVA are assumed identical, that means $\alpha_{j}=\alpha$ and $\zeta_{j}=\zeta(j=1,2, \ldots, n)$. The other parameters in Eq. (8) are described as

$$
\begin{aligned}
& A=4 \lambda^{4} n^{2} \beta^{2} \alpha^{2} \\
& B=\left(\eta^{2} \beta^{2}-n \alpha^{2} \gamma^{2}\right)^{2} \\
& C=4 n^{2} \alpha^{2} \lambda^{4} \beta^{2}\left[\left(\mu \eta^{2} \beta^{2}+\beta^{2}-1\right)^{2}+4 \beta^{2} \zeta_{s}^{2}\right], \\
& D=8 \zeta_{s} \beta^{2} \lambda^{2} n \alpha\left[\left(\eta^{2} \beta^{2}-n \alpha^{2} \gamma^{2}\right)\left(\mu \eta^{2} \beta^{2}+\beta^{2}-1\right)+\eta^{2} \beta^{2}\left(n \mu \alpha^{2} \gamma^{2}-\beta^{2}+1\right)+n \alpha^{2} \gamma^{2}\left(\beta^{2}-1\right)\right] \\
& E=\left[n \mu \alpha^{2} \eta^{2} \gamma^{2} \beta^{2}+\left(1-\beta^{2}\right)\left(\eta^{2} \beta^{2}-n \alpha^{2} \gamma^{2}\right)\right]^{2}+4 \beta^{2} \zeta_{s}^{2}\left(\eta^{2} \beta^{2}-n \alpha^{2} \gamma^{2}\right)^{2} .
\end{aligned}
$$

To reduce torsional vibration of damped rotary system, the parameters $\alpha$ and $\zeta$ are needed to define for minimum torsional angle $\theta_{s}$ (Eq. (4)) at resonant frequency. These 
parameters are called optimal parameters of DVAs (signed $\alpha_{\text {opt }}$ and $\zeta_{\text {opt }}$ ) that will be determined in the below section.

\section{OPTIMAL PARAMETER OF DYNAMIC VIBRATION ABSORBER}

When the rotary system is coupled with a damper, the fixed-points feature is diminished. However, the amplitude magnification curves roughly pass two points when the rotary system is lightly or moderately damped, and the mass ratio between the rotary system and the DVA is small. To satisfying the above-mentioned conditions, it is assumed that the fixed-point theory is approximately maintained [28]. Based on this assumption, an approximate solution for the optimum tuning parameter $\alpha_{\text {opt }}$ for the damped model can be realized. The two approximated fixed points (signed as $S$ and $T$ ) are found by finding the intersections of the amplitude magnification curves. The two AMF curves defined at $\zeta$ equals to 0 , and $\zeta$ approaches $\infty$ are chosen

$$
\begin{gathered}
\left.H\right|_{\zeta=0}=\sqrt{\frac{B}{E}}=\sqrt{\frac{\left(\eta^{2} \beta^{2}-n \alpha^{2} \gamma^{2}\right)^{2}}{\left[n \mu \alpha^{2} \eta^{2} \gamma^{2} \beta^{2}+\left(1-\beta^{2}\right)\left(\eta^{2} \beta^{2}-n \alpha^{2} \gamma^{2}\right)\right]^{2}+4 \beta^{2} \zeta_{s}^{2}\left(\eta^{2} \beta^{2}-n \alpha^{2} \gamma^{2}\right)^{2}}}, \\
\left.H\right|_{\zeta \rightarrow \infty}=\lim _{\zeta \rightarrow \infty} \sqrt{\frac{A \zeta^{2}+B}{C \zeta^{2}+D \zeta+E}}=\sqrt{\frac{A}{C}}=\sqrt{\frac{1}{\left(\mu \eta^{2} \beta^{2}+\beta^{2}-1\right)^{2}+4 \beta^{2} \zeta_{s}^{2}}}
\end{gathered}
$$

Equating $H$ in Eqs. (10) and (11) results in

$$
\left|\frac{\left(\eta^{2} \beta^{2}-n \alpha^{2} \gamma^{2}\right)^{2}}{\left[n \mu \alpha^{2} \eta^{2} \gamma^{2} \beta^{2}+\left(1-\beta^{2}\right)\left(\eta^{2} \beta^{2}-n \alpha^{2} \gamma^{2}\right)\right]^{2}+4 \beta^{2} \zeta_{s}^{2}\left(\eta^{2} \beta^{2}-n \alpha^{2} \gamma^{2}\right)^{2}}\right|=\left|\frac{1}{\left(\mu \eta^{2} \beta^{2}+\beta^{2}-1\right)^{2}+4 \beta^{2} \zeta_{s}^{2}}\right| .
$$

Solving Eq. (12) gives the frequency ratios at $S$ and $T$ as

$$
\beta_{S, T}^{2}=\frac{n \alpha^{2} \gamma^{2}\left(\mu \eta^{2}+1\right)+\eta^{2} \mp \sqrt{n^{2} \alpha^{4} \gamma^{4}(\mu \eta+1)^{2}+\eta^{2}\left(\eta^{2}-2 n \alpha^{2} \gamma^{2}\right)}}{\eta^{2}\left(\eta^{2} \mu+2\right)} .
$$

To find the optimal tuning ratio, let the ordinates of points $S$ and $T$ be equal resulting in

$$
\frac{1}{\left|\left(\mu \eta^{2} \beta_{S}^{2}+\beta_{S}^{2}-1\right)^{2}+4 \beta_{S}^{2} \zeta_{S}^{2}\right|}=\frac{1}{\left|\left(\mu \eta^{2} \beta_{T}^{2}+\beta_{T}^{2}-1\right)^{2}+4 \beta_{T}^{2} \zeta_{S}^{2}\right|} .
$$

By substituting $\beta_{S}, \beta_{T}$ in Eq. (13) into Eq. (14), and then solving this equation, $\alpha_{o p t}$ is found as follows

$$
\alpha_{o p t}=\frac{\eta}{\gamma \sqrt{n}\left(1+\eta^{2} \mu\right)} \sqrt{1-2 \zeta_{s}^{2}-\frac{2 \zeta_{s}^{2}}{\left(\eta^{2} \mu+1\right)}} .
$$

From Eq. (15), the optimal tuning parameter of undamped system can be calculated by setting the damping ratio of the main system $\zeta_{s}=0$. Then resulting $\alpha_{o p t}$ for undamped system is

$$
\alpha_{o p t}=\frac{\eta}{\gamma \sqrt{n}\left(1+\eta^{2} \mu\right)}
$$


It is seen that $\alpha_{\text {opt }}$ in Eq. (16) is the same as the one derived for undamped system by using the fixed-point theory [25,26]. Phuc et al. [26] found the optimal tuning parameter of DVA for damped rotary system using an equivalent undamped model as following

$$
\alpha_{o p t}=\frac{\eta}{\gamma \sqrt{n}\left(1+\eta^{2} \mu\right)}\left(\sqrt{\frac{4 \zeta_{s}^{2}}{\pi^{2}}+1}-\frac{2 \zeta_{s}}{\pi}\right) .
$$

The comparison between the optimal parameter proposed in this paper and [26] will be presented in the next section.

Fig. 2 shows the AMF curves versus $\beta$ with varying damping ratios of DVA $(\zeta)$. To reduce the maximum peaks of AMF, we determine the value of $\zeta$ so that the AMF function has two equal peak values with a minimal distance from a straight line $L$ as shown in Fig. 2.

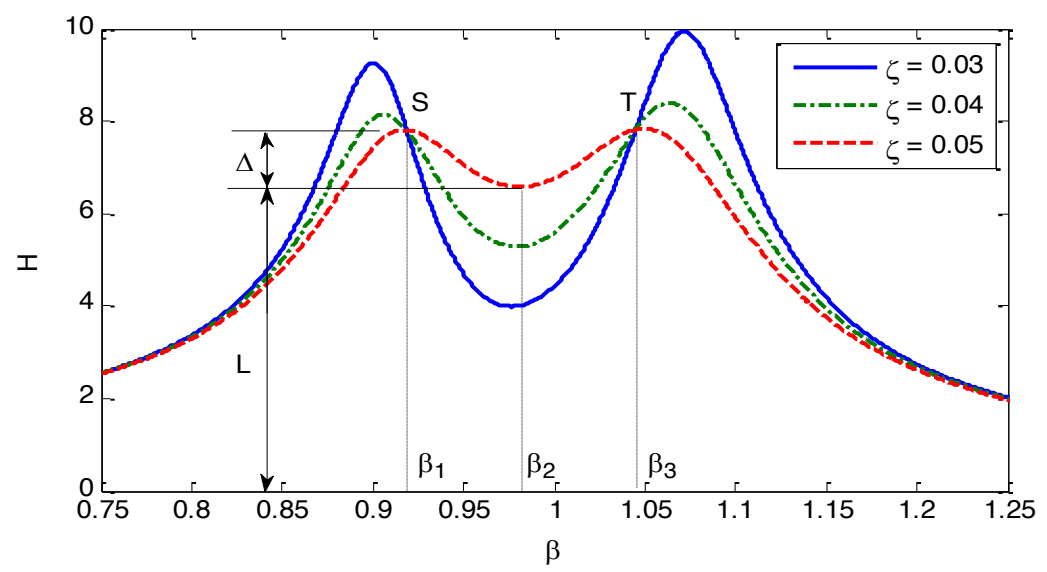

Fig. 2. Demonstration of parameters in Eq. (18)

In this way, the optimum solution can be found by using the Chebyshev equioscillation theorem $[18,19]$. To this end, the following equations will be solved

$$
\left\{\begin{array}{l}
\left.\frac{d H}{d \beta}\right|_{\beta=\beta_{1}}=0,\left.\frac{d H}{d \beta}\right|_{\beta=\beta_{2}}=0,\left.\frac{d H}{d \beta}\right|_{\beta=\beta_{3}}=0, \\
H\left(\beta_{1}\right)-H\left(\beta_{3}\right)=0,2 L-\left[H\left(\beta_{1}\right)+H\left(\beta_{3}\right)\right]=0,2 \Delta-\left[H\left(\beta_{1}\right)-H\left(\beta_{2}\right)\right]=0 .
\end{array}\right.
$$

where $\Delta$ indicates the maximum peak value of the AMF curve determined from the ordinate $H=L$ (see Fig. 2). $\beta_{1}, \beta_{2}$ and $\beta_{3}$ are the frequency ratios at which the AMF curve reaches maximum and minimum. Unlike the result of Ghosh and Basu [28], which only found the optimal tuning parameter, this study allows finding the optimal damping parameter through solving the system of 6 nonlinear equations (18) for 6 unknowns (i.e. $\beta_{1}, \beta_{2}, \beta_{3}, L, \Delta$ and $\zeta$ ). Compared to the system of equations of Liu and Coppola [19], the unknown $\alpha$ is eliminated in our system of equations (18). This is because the expression of the optimal tuning parameter (15) has been substituted into the amplitude magnification factor $(H)$ before the equation system equations (18) were established. 
The fsolve function provided by Matlab is used to solve system equations (18). With respect to the nonlinear equations system, the fsolve solver requires good initial values of the roots for a quick convergence. The initial parameters were set as: $\beta_{1}=0.85, \beta_{2}=$ $1, \beta_{3}=1.05, L=0, \Delta=0$. The initial value of $\zeta$ is selected as the optimal value for undamped rotary system, that is

$$
\zeta=\sqrt{\frac{3}{8} \frac{\mu \eta^{4} \gamma^{2}}{n \lambda^{4}\left(1+\mu \eta^{2}\right)}} .
$$

The optimal damping ratio of undamped in Eq. (19) is determined by using the fixedpoint theory $[25,26]$.

\section{NUMERICAL RESULTS AND DISCUSSION}

To demonstrate the proposed method, this section presents numerical simulation for a sample damped DVA system with the parameters given in Tab. 1. The parameters of this system are taken from reference [26]. First, a comparison is performed to compare the current method with the existing method. Then, further simulations are carried out to examine the performance of the optimal DVA and investigate the effect of several important parameters of DVA system on the optimal parameters.

\subsection{Model comparison}

The present method is compared with the method proposed by Phuc et al. [26]. In their method [26], the authors obtained the optimal parameters of damped DVA system via two steps. In the first step, the damped rotary system is converted into an equivalent undamped rotary system using the least squares estimation of equivalent linearization method, which was developed by Anh et al. [27]. The second step determines the optimal parameters of DVA of equivalent undamped rotary system using the traditional fixed point theory.

Tab. 2 and Fig. 3 show the effect of damping ratio of rotary system $\zeta_{s}$ on the optimal parameters determined by present method and the method proposed in [26]. The optimal parameters are determined for two values of mass ratio, $\mu=0.033$ and $\mu=0.05$. Fig. 3(a) shows that increasing $\zeta_{s}$ leads to the reduction of optimal tuning parameter of

Table 1. Input parameters of damped rotary system and DVA

\begin{tabular}{lcc}
\hline \multicolumn{1}{c}{ Parameters } & Unit & Value \\
\hline Mass of main disk $\left(m_{s}\right)$ & $\mathrm{kg}$ & 6 \\
Gyration radius of main disk $\left(\rho_{s}\right)$ & $\mathrm{m}$ & 0.12 \\
Amplitude of excitation moment $\left(M_{0}\right)$ & $\mathrm{Nm}$ & 8.0 \\
Stiffness of main spring $\left(k_{s}\right)$ & $\mathrm{Nm} / \mathrm{rad}$ & 12,000 \\
Gyration radius of passive disk $\left(\rho_{a}\right)$ & $\mathrm{m}$ & 0.12 \\
Radial position of dampers of DVA $\left(e_{2}\right)$ & $\mathrm{m}$ & 0.08 \\
Radial position of springs of DVA $\left(e_{1}\right)$ & $\mathrm{m}$ & 0.05 \\
Number of springs and dampers of DVA $(n)$ & - & 4 \\
\hline
\end{tabular}


DVA. Higher $\mu$ value requires a smaller $\alpha_{o p t}$ value for both methods. On contrary, $\zeta_{\text {opt }}$ calculated by the present method increases with increasing either $\zeta_{s}$ or $\mu$. The value of $\zeta_{\text {opt }}$ found by [26] is almost constant in entire range of $\zeta_{s}$. Fig. 3 shows that the optimal parameters of the present method and [26] are only approximated at low $\zeta_{s}$.

Table 2. Optimum tuning ratio and damping ratio of DVA for different rotary system damping ratios

\begin{tabular}{ccccccccc}
\hline \multirow{2}{*}{$\begin{array}{c}\text { Rotary system } \\
\text { damping ratios }\end{array}$} & \multicolumn{3}{c}{ Optimum tuning ratio $\left(\alpha_{\text {opt }}\right)$} & \multicolumn{3}{c}{ Optimum damping ratio $\left(\zeta_{\text {opt }}\right)$} \\
\cline { 2 - 9 }$\left(\zeta_{s}\right)$ & \multicolumn{2}{c}{ Phuc et al. [26] } & \multicolumn{2}{c}{ Present study } & \multicolumn{2}{c}{ Phuc et al. [26] } & \multicolumn{2}{c}{ Present study } \\
\cline { 2 - 9 } & $\mu=0.033$ & $\mu=0.05$ & $\mu=0.033$ & $\mu=0.05$ & $\mu=0.033$ & $\mu=0.05$ & $\mu=0.033$ & $\mu=0.05$ \\
\hline 0.010 & 1.1539 & 1.1356 & 1.1611 & 1.1426 & 0.0516 & 0.0626 & 0.0527 & 0.0641 \\
0.015 & 1.1503 & 1.1320 & 1.1608 & 1.1424 & 0.0516 & 0.0626 & 0.0530 & 0.0644 \\
0.020 & 1.1466 & 1.1284 & 1.1604 & 1.1420 & 0.0516 & 0.0626 & 0.0532 & 0.0648 \\
0.025 & 1.1430 & 1.1248 & 1.1599 & 1.1415 & 0.0516 & 0.0626 & 0.0539 & 0.0654 \\
0.030 & 1.1393 & 1.1212 & 1.1592 & 1.1408 & 0.0516 & 0.0626 & 0.0546 & 0.0658 \\
0.035 & 1.1357 & 1.1177 & 1.1585 & 1.1401 & 0.0516 & 0.0626 & 0.0559 & 0.0662 \\
0.040 & 1.1321 & 1.1141 & 1.1576 & 1.1393 & 0.0516 & 0.0626 & 0.0580 & 0.0672 \\
\hline
\end{tabular}

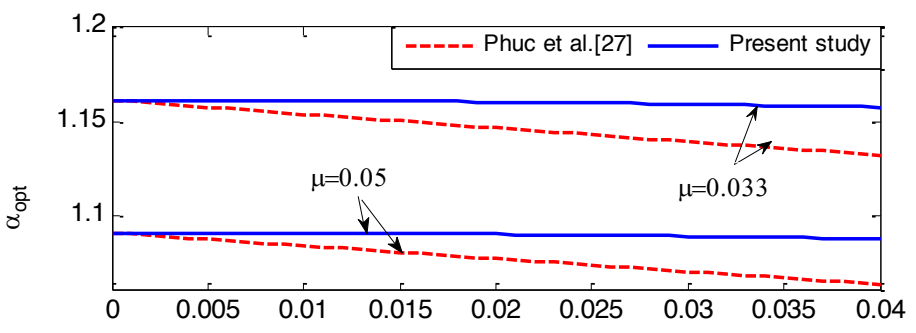

(a)

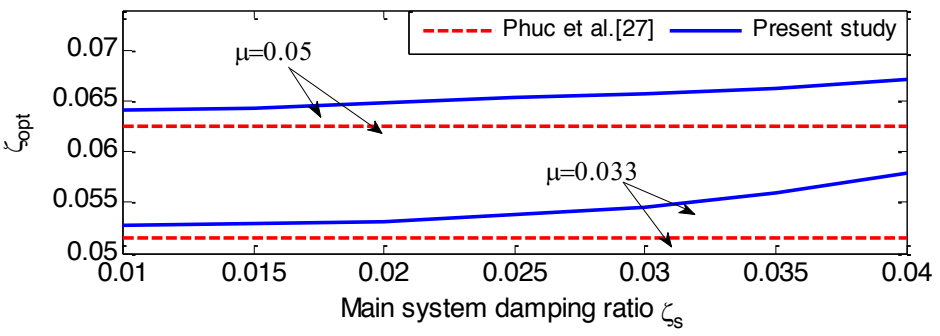

(b)

Fig. 3. Effect of main system damping ratio and mass ratio on optimal parameters

Fig. 4 shows the amplitude amplification factor determined by the present method and [26] for several values of $\zeta_{s}$. There are two peaks of the AMF, which occur around the resonance frequency.

It can be seen from Fig. 4(a) that at low damping ratio such as $\zeta_{s}=0.01$, the AMF calculated by the two methods are almost the same. This is because the values of optimum parameters obtained by the two methods at low damping ratios are approximated 


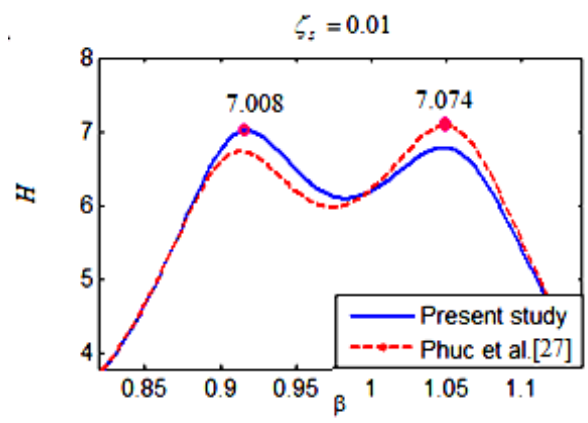

(a)

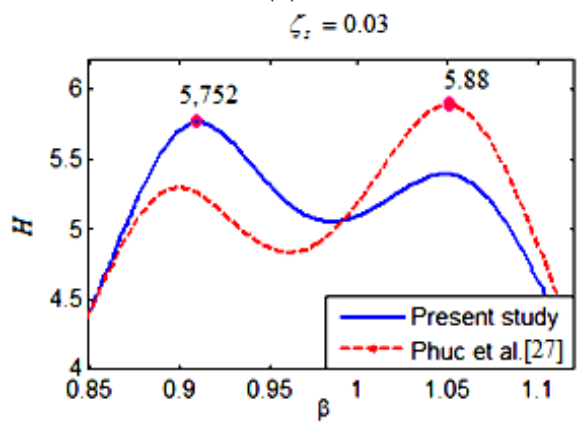

(c)

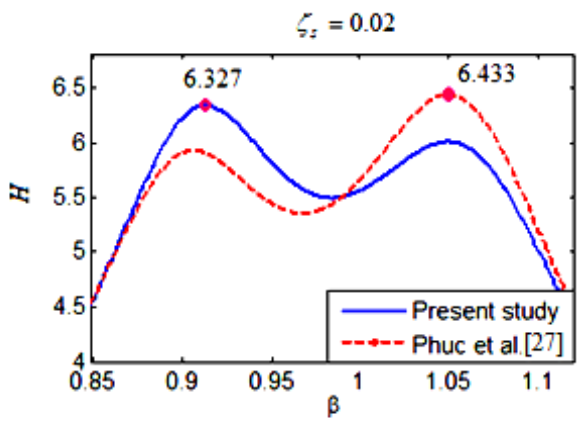

(b)

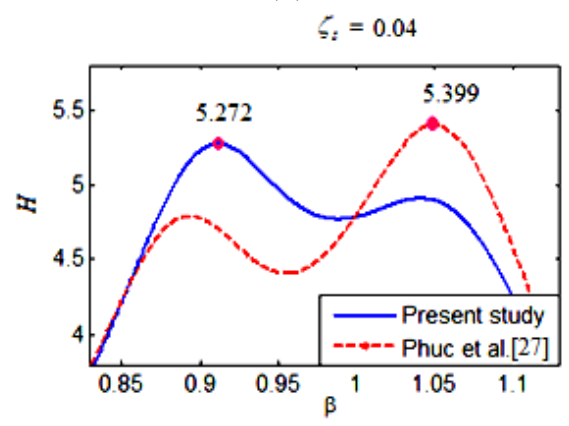

(d)

Fig. 4. Comparison of the AMF by present method and [26] with varying damping ratios $(\mu=0.033)$

as shown in Fig. 3. When the damping ratio increases, the maximal peak value of AMF determined by [26] is higher than that of the present method (show in Fig. 4(d)).

Tab. 3 shows the AFM calculated at the resonant frequency $\left(H_{(\beta=1)}\right)$ by the present method and [26] with varying the damping ratio. This table also shows the maximum AFM $\left(H_{\max }\right)$ calculated by the two methods. From this table, the maximum reduction percentages of $H$ estimated at $\beta=1$, and $H_{\max }$ are $1.89 \%$ and $5.24 \%$, respectively. Therefore, present method clearly shows better vibration suppression compared to [26].

Fig. 5 compares the maximum of AMF determined by proposed formulae and [26] with varying the damping ratio of the damped rotary system. It can be seen that the increase rate of difference in maximal peaks of AMF is increased as $\zeta_{s}$ is greatly increased. Therefore, the proposed optimal parameters give a better mitigation than that of [26] in terms of maximum magnification factor.

The sensitivity of optimal parameters on the amplitude magnification factor is presented in Fig. 6. In which, the vertical axes show the deviation percentage of AMF, which are calculated by

$$
\Delta H_{\zeta}=\frac{H_{\zeta, \alpha_{o p t}}-H_{\alpha_{o p t}, \zeta_{o p t}}}{H_{\alpha_{o p t}, \zeta_{o p t}}} \times 100
$$


Table 3. Comparison of AFM estimated at resonance frequency $\left(H_{(\beta=1)}\right)$ and the maximal value of $\mathrm{AFM}\left(H_{\max }\right)$ with varying the damping ratio

\begin{tabular}{ccccccc}
\hline \multirow{2}{*}{$\begin{array}{c}\text { Rotary } \\
\text { system } \\
\text { damping } \\
\text { ratios } \\
\left(\zeta_{s}\right)\end{array}$} & \multicolumn{3}{c}{$H$ in resonance frequency } & \multicolumn{3}{c}{$H_{\max }$ in resonance frequency region } \\
\cline { 2 - 7 } & al. [26] & $\begin{array}{c}\text { Present } \\
\text { study }\end{array}$ & $\begin{array}{c}\text { Improvement from Phuc's } \\
\text { result in percentage terms }\end{array}$ & $\begin{array}{c}\text { Phuc et } \\
\text { al. [26] }\end{array}$ & $\begin{array}{c}\text { Present } \\
\text { study }\end{array}$ & $\begin{array}{c}\text { Improvement from Phuc's } \\
\text { result in percentage terms }\end{array}$ \\
\hline 0.010 & 6.227 & 6.200 & 0.43 & 7.074 & 7.008 & $\mu=0.033$ \\
0.015 & 5.926 & 5.867 & 1.00 & 6.740 & 6.653 & 0.93 \\
0.020 & 5.657 & 5.561 & 1.70 & 6.443 & 6.327 & 1.29 \\
0.025 & 5.414 & 5.318 & 1.77 & 6.145 & 6.029 & 1.80 \\
0.030 & 5.194 & 5.096 & 1.89 & 5.880 & 5.572 & 5.24 \\
0.035 & 4.993 & 4.920 & 1.46 & 5.631 & 5.501 & 2.31 \\
0.040 & 4.810 & 4.790 & 0.42 & 5.399 & 5.272 & 2.35 \\
\hline
\end{tabular}

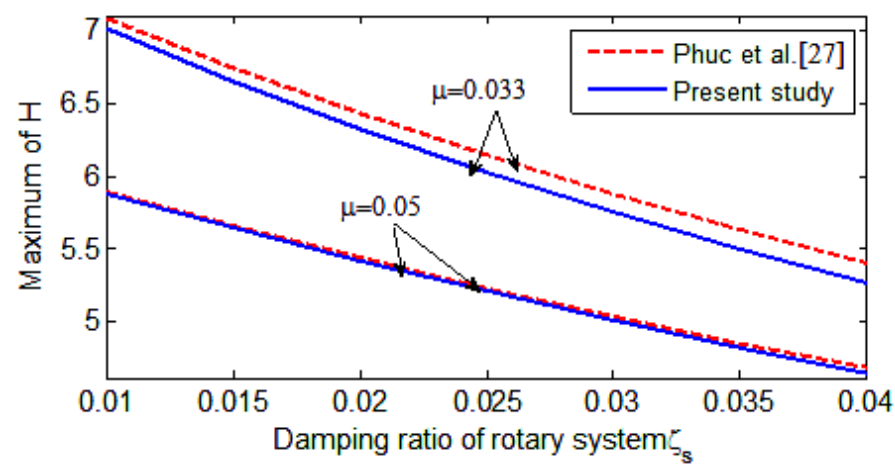

Fig. 5. The effect of damping ratio of main structure on maximum of $\operatorname{AMF}(\mu=0.033)$

and

$$
\Delta H_{\alpha}=\frac{H_{\alpha, \zeta_{o p t}}-H_{\alpha_{o p t}, \zeta_{o p t}}}{H_{\alpha_{o p t}, \zeta_{o p t}}} \times 100,
$$

where $H_{\alpha, \zeta_{o p t}}, H_{\zeta, \alpha_{o p t}}$ and $H_{\alpha_{o p t}, \zeta_{o p t}}$ are the maximum values of AMF determined at the correspondent damping and tuning ratios given in the subscript indexes.

Fig. 6 demonstrates that the tuning parameter is more sensitive to the variation of AMF than the damping ratio. When the damping ratio varies from $-10 \%$ to $40 \%$ around $\zeta_{\text {opt }}$, the maximum deviation percentage of AMF $\left(\Delta H_{\zeta}\right)$ is below $1 \%$, whereas $\Delta H_{\zeta}$ is higher than $40 \%$ in the case of error in $\alpha_{o p t}$. In addition, the actual optimal damping ratio should be about $15 \%$ higher than the calculated value with Eq. (19). In the case of tuning ratio, the actual optimal tuning ratio should be about $1.5 \%$ smaller than the value found by proposed equation (15). However, the differences between $\Delta H_{\zeta}$ and $\Delta H_{\alpha}$ computed by actual optimal parameters and proposed formulae (Eqs. (20) and (21)) are relatively small (about $2 \%$ ). Therefore, the proposed optimal parameters are approximated solutions, although they can still be used with satisfactory accuracy. Fig. 6 also shows that the 

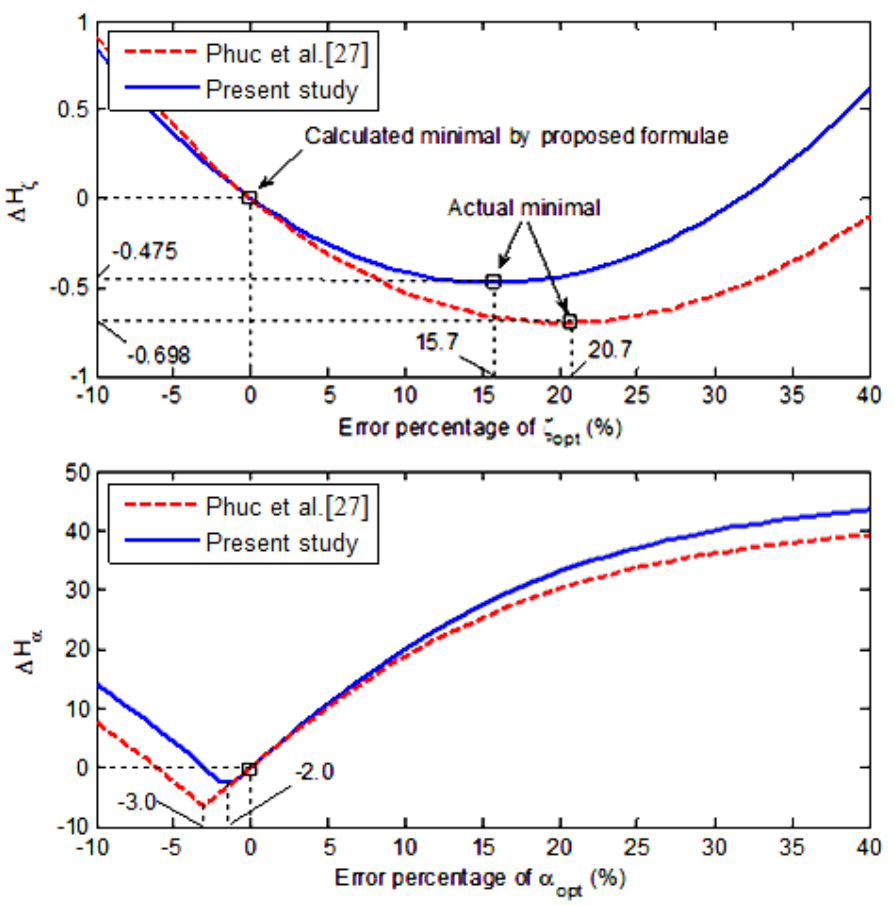

Fig. 6. Effect of error in optimal parameters on AMF $\left(\zeta_{s}=0.1, \mu=0.033\right)$

actual optimal damping and tuning ratios should be around $20 \%$ higher and $3 \%$ lower than those proposed in [26], respectively. Therefore, the proposed optimal parameters in this paper are closer to actual optimality point than those in [26].

\subsection{Numerical investigation}

In this section, numerical simulation is performed to examine the effect optimal DVA on the system vibration. Moreover, the effects of damping ratio of rotary system and mass ratio on the optimal parameters of DVA are investigated. The parameters of the damped rotary system are the same as those depicted in Tab. 1.

Fig. 7 shows the AMF and vibration response in time domain of the damped rotary system without DVA and with optimally designed DVA.

Fig. 7(a) shows that using optimal DVA remarkably reduces the peak of AMF around the resonance frequency. At the frequencies far from the resonance, such as at $\beta=0.85$, the AMF may not be reduced. In general, vibration control using passive DVAs is normally very effective for a certain frequency-bandwidth. However, the vibration of the system is usually large around the resonant frequency (see Fig. 7(a)). Hence, the effectiveness of optimal DVA is still well achieved.

The vibration response in time domain in Fig. $7(\mathrm{~b})$ is simulated at the frequency ratio $\beta=1$ (resonance condition). It is clear that the rotary system attached with optimal DVA quickly obtains the steady state vibration with vibration amplitude significant lower 


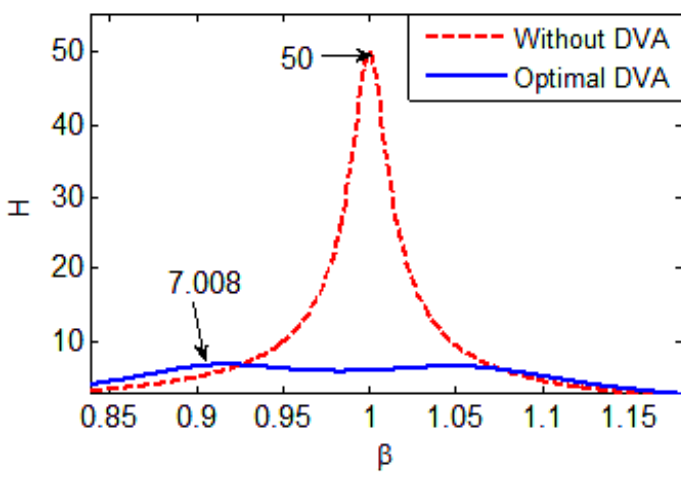

(a)

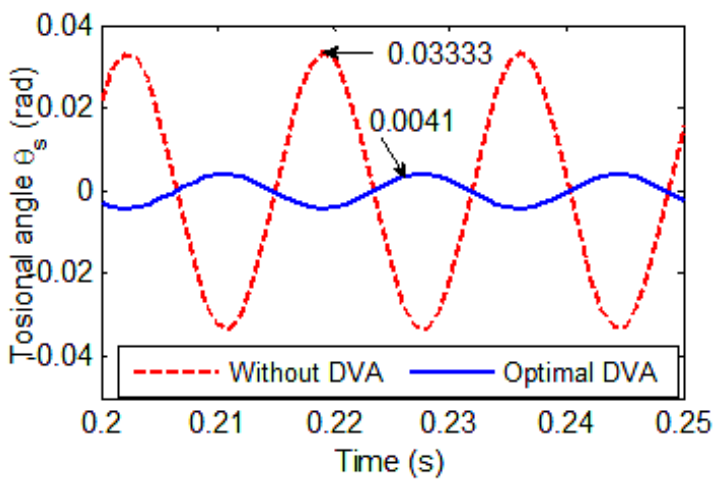

(b)

Fig. 7. Performance of the system without DVA and with optimal DVA, (a) AMF, (b) vibration response in time domain $\left(\Omega=\omega_{s}, \zeta_{s}=0.01, \mu=0.0333, n=4\right)$

than that without DVA, the effective of vibration control to $87.6 \%$ in resonance condition (Fig. 7(b)).

Fig. 8 shows the deviation percentage of AMF calculated by Eqs. (20) and (21) for several values of $\zeta_{s}$. Fig. 8(a) shows that increasing $\zeta$ results in a larger difference between the actual and predicted optimal damping ratio (signed as $\Delta \zeta_{\text {opt }}$ ). Such as, $\Delta \zeta_{\text {opt }}$ is about $2.7 \%$ for $\zeta_{s}=0.02$, and $\Delta \zeta_{\text {opt }}$ is approximate $17 \%$ for $\zeta_{s}=0.1$.

The difference between the actual and predicted optimal tuning ratio (signed as $\left.\Delta \alpha_{o p t}\right)$ is also increased with the increase of $\zeta_{s}$ as shown in Fig. 8(b). However, the value of $\Delta \alpha_{o p t}$ is relatively small (less than $2 \%$ ) even in the case of highest damping ratio $\left(\zeta_{s}=0.1\right)$. From Fig. 8, the maximum differences of $\Delta H_{\zeta}$ and $\Delta H_{\alpha}$ determined at actual optimal parameters and proposed formulae are around $0.453 \%$ and $3.622 \%$, respectively.

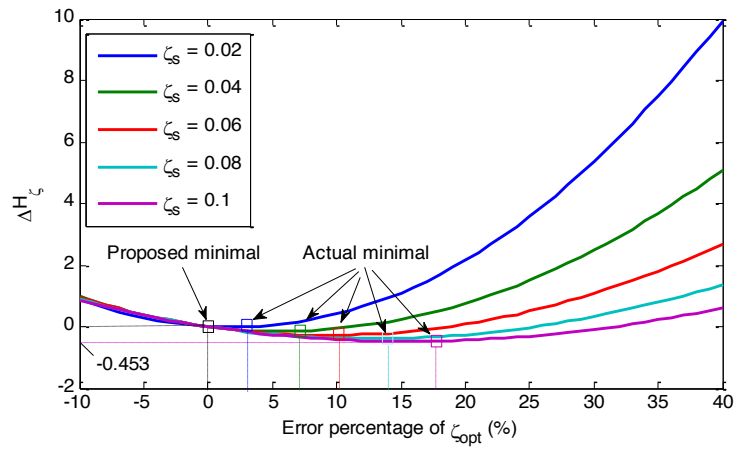

(a)

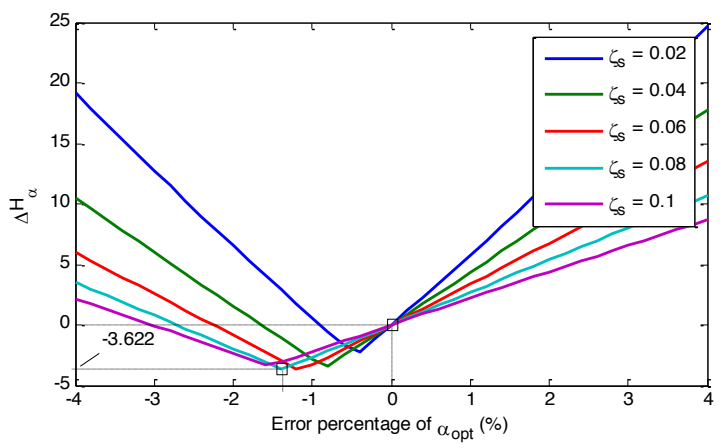

(b)

Fig. 8. Effect of error in optimal parameters on AMF for different $\zeta_{s}(\mu=0.033)$

Fig. 9 shows the deviation percentage of AMF with varying $\mu$ and it illustrates that the sensitivity of optimal parameters is dependent on $\mu$. In particularly, the sensitivity of 

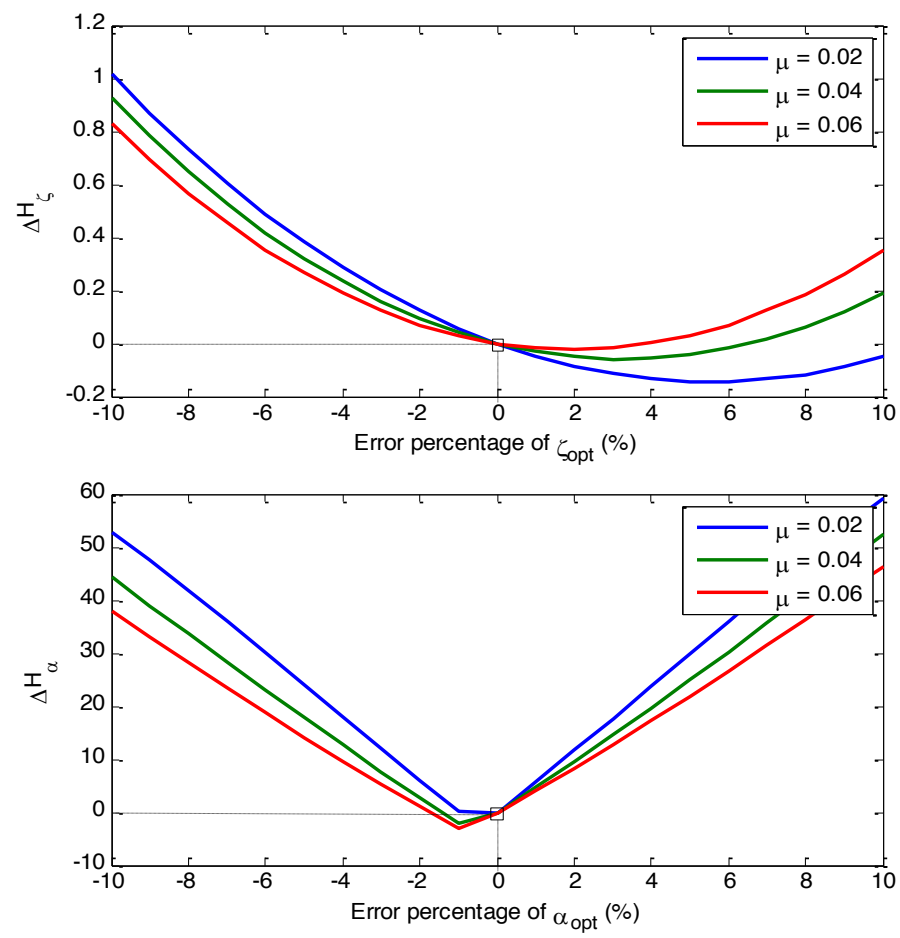

Fig. 9. Effect of error in optimal parameters on the AMF for different mass ratios $\left(\zeta_{s}=0.03\right)$

$\alpha_{\text {opt }}$ is reduced with the increase of $\mu$. For example, with the same $10 \%$ erroneous in $\alpha_{\text {opt }}$, $\Delta H_{\alpha}$ reduces from $59.21 \%$ to $46.43 \%$ when $\mu$ is increased from 0.02 to 0.06 . It is seen that the values of $\Delta H_{\alpha}$ and $\Delta H_{\zeta}$ determined at actual optimal parameters vary with varying $\mu$; however, the changes are minor.

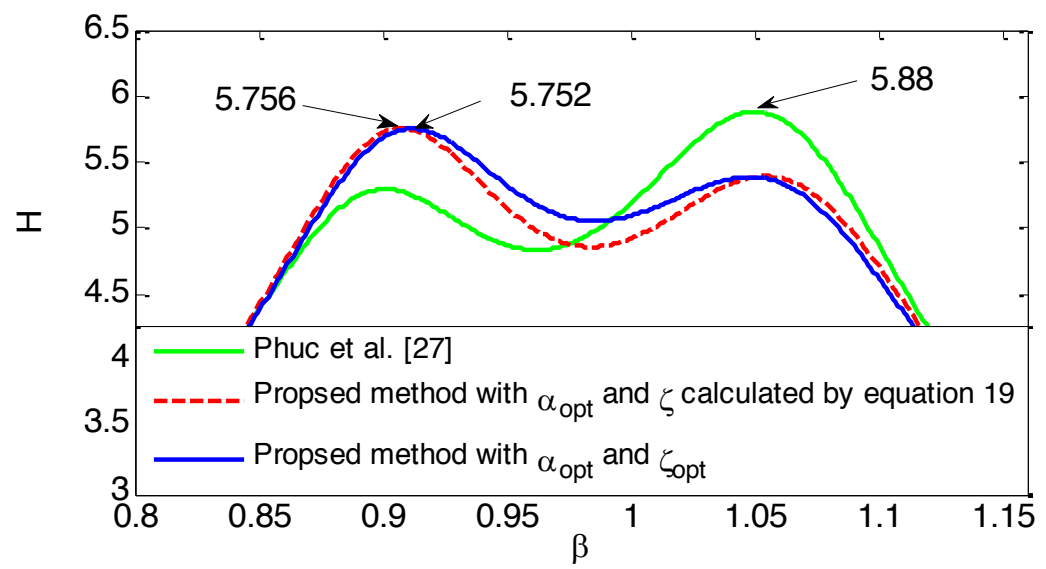

Fig. 10. Comparison of the AMF determined by the proposed method and [26] with $\left(n=4, \mu=0.033, \zeta_{s}=0.03\right)$ 
Fig. 10 shows the comparison of the AMF of a damped rotary system determined by the method in [26] and the proposed method in this study. In this figure, the solid green line indicates the AMF determined by [26] and the solid blue line presents the AMF calculated with optimal tuning and damping ratios determined by the present method. It can be seen that the maximum peak value of AMF curve obtained by the proposed method is smaller than that by [26]. Fig. 10 further shows the AMF estimated with the optimal tuning ratio proposed in this study, while a non-optimal damping ratio is used as calculated by Eq. (19). The AFM curve for this case is indicated by the red-dashed line in Fig. 10. It is observed that even using only the proposed optimal tuning ratio, the maximum value of the AMF curve is still smaller than that of [26]. Hence, the AMF by the proposed method is a generally superior compared to [26].

\section{CONCLUSIONS}

In this study, the optimum parameters of a DVA attached to a damped rotary system are proposed. The aim of optimization is to minimize the maximum amplitude magnification factor of the damped rotary system. A closed-form formula for the optimum tuning ratio is obtained using the fixed-point theory with assuming low-to-moderate damping in the damped rotary system. A semi-analytical process for calculating the optimal damping ratio of DVA is presented. The numerical results prove that the proposed optimal parameters have better suppression of the resonant vibration amplitude than the existing method and the control performance of DVA can be up to $90 \%$. A time history analysis is performed to demonstrate the efficiency of the proposed formulae. It is shown that there is a slight difference between actual and proposed optimal tuning and damping ratios of DVA. Therefore, the proposed optimal formulation is an approximate solution. Nevertheless, the error caused by approximated solution is very small as shown by $3.62 \%$ difference in deviation percentage of magnification factor (Fig. 8).

\section{ACKNOWLEDGMENT}

This research is funded by Hung Yen University of Technology and Education, Vietnam.

\section{REFERENCES}

[1] T. Asami, Y. Mizukawa, and T. Ise. Optimal design of double-mass dynamic vibration absorbers minimizing the mobility transfer function. Journal of Vibration and Acoustics, 140, (6), (2018). https://doi.org/10.1115/1.4040229.

[2] H. Frahm. Device for damped vibration of bodies. U.S. Patent No. 989958, (1909).

[3] J. Ormondroyd and J. P. Den Hartog. The theory of the dynamic vibration absorber. Trans. ASME, Applied Mechanics, 50, (1928), pp. 9-22.

[4] J. P. Den Hartog. Mechanical vibrations. McGraw-Hill, New York, (1934).

[5] J. Dayou. Fixed-points theory for global vibration control using vibration neutralizer. Journal of Sound and Vibration, 292, (3-5), (2006), pp. 765-776. https://doi.org/10.1016/j.jsv.2005.08.032. 
[6] K. Liu and J. Liu. The damped dynamic vibration absorbers: revisited and new result. Journal of Sound and Vibration, 284, (3-5), (2005), pp. 1181-1189. https://doi.org/10.1016/j.jsv.2004.08.002.

[7] H. E. Kalehsar and N. Khodaie. Optimization of response of a dynamic vibration absorber forming part of the main system by the fixed-point theory. KSCE Journal of Civil Engineering, 22, (7), (2018), pp. 2354-2361. https://doi.org/10.1007/s12205-017-1237-y.

[8] T. Asami, O. Nishihara, and A. M. Baz. Analytical solutions to $H_{\infty}$ and $H_{2}$ optimization of dynamic vibration absorbers attached to damped linear systems. Journal of Vibration and Acoustics, 124, (2), (2002), pp. 284-295. https://doi.org/10.1115/1.1456458.

[9] T. Asami and O. Nishihara. $H_{2}$ optimization of the three-element type dynamic vibration absorbers. Journal of Vibration and Acoustics, 124, (4), (2002), pp. 583-592. https://doi.org/10.1299/kikaic.68.76.

[10] O. Nishihara and T. Asami. Closed-form solutions to the exact optimizations of dynamic vibration absorbers (minimizations of the maximum amplitude magnification factors). Journal of Vibration and Acoustics, 124, (4), (2002), pp. 576-582. https://doi.org/10.1115/1.1500335.

[11] Y. Shen, X. Wang, S. Yang, and H. Xing. Parameters optimization for a kind of dynamic vibration absorber with negative stiffness. Mathematical Problems in Engineering, 2016, (2016). https://doi.org/10.1155/2016/9624325.

[12] S. H. Crandall and W. D. Mark. Random vibration in mechanical systems. Academic Press, London, (1963).

[13] M. Zilletti, S. J. Elliott, and E. Rustighi. Optimisation of dynamic vibration absorbers to minimise kinetic energy and maximise internal power dissipation. Journal of Sound and Vibration, 331, (18), (2012), pp. 4093-4100. https://doi.org/10.1016/j.jsv.2012.04.023.

[14] H. Yamaguchi. Damping of transient vibration by a dynamic absorber. Trans. Jpn. Soc. Mech. Eng. Ser. C, 54, (499), (1988), pp. 561-568. https://doi.org/10.1299/kikaic.54.561.

[15] T. Argentini, M. Belloli, and P. Borghesani. A closed-form optimal tuning of mass dampers for one degree-of-freedom systems under rotating unbalance forcing. Journal of Vibration and Acoustics, 137, (3), (2015). https://doi.org/10.1115/1.4029576.

[16] P. Bisegna and G. Caruso. Closed-form formulas for the optimal pole-based design of tuned mass dampers. Journal of Sound and Vibration, 331, (10), (2012), pp. 2291-2314. https://doi.org/10.1016/j.jsv.2012.01.005.

[17] A. G. Thompson. Optimizing the untuned viscous dynamic vibration absorber with primary system damping-a frequency locus method-reply. Journal of Sound and Vibration, 73, (3), (1980), pp. 469-472. https://doi.org/10.1016/0022-460X(80)90528-3.

[18] E. Pennestri. An application of Chebyshev's min-max criterion to the optimal design of a damped dynamic vibration absorber. Journal of Sound and Vibration, 217, (4), (1998), pp. 757765. https://doi.org/10.1006/jsvi.1998.1805.

[19] K. Liu and G. Coppola. Optimal design of damped dynamic vibration absorber for damped primary systems. Transactions of the Canadian Society for Mechanical Engineering, 34, (1), (2010), pp. 119-135. https://doi.org/10.1139/tcsme-2010-0008.

[20] N. V. Khang, T. Q. Loc, and N. A. Tuan. Parameter optimization of tuned mass damper for three-degree-of-freedom vibration systems. Vietnam Journal of Mechanics, 35, (3), (2013), pp. 215-224. https://doi.org/10.15625/0866-7136/35/3/3315.

[21] N. D. Anh and N. C. Sang. On the optimal control force applied to tuned mass dampers for multi-degree-of-freedom systems. Vietnam Journal of Mechanics, 26, (1), (2004), pp. 1-10. https://doi.org/10.15625/0866-7136/26/1/5682. 
[22] N. D. Anh, N. H. Hai, N. X. Nguyen, D. V. Hieu, and P. T. T. My. Weighted averaging technique for the design of dynamic vibration absorber installed in damped primary structures. Vietnam Journal of Mechanics, 40, (2), (2018), pp. 155-162. https://doi.org/10.15625/0866$7136 / 10732$.

[23] L. Murawski and A. Charchalis. Simplified method of torsional vibration calculation of marine power transmission system. Marine Structures, 39, (2014), pp. 335-349. https://doi.org/10.1016/j.marstruc.2014.10.004.

[24] G. Wenzhi and H. Zhiyong. Active control and simulation test study on torsional vibration of large turbo-generator rotor shaft. Mechanism and Machine Theory, 45, (9), (2010), pp. 13261336. https://doi.org/10.1016/j.mechmachtheory.2010.04.005.

[25] X.-T. Vu, D.-C. Nguyen, D.-D. Khong, and V.-C. Tong. Closed-form solutions to the optimization of dynamic vibration absorber attached to multi-degrees-of-freedom damped linear systems under torsional excitation using the fixed-point theory. Journal of Multi-body Dynamics, 232, (2), (2018), pp. 237-252. https://doi.org/10.1177/1464419317725216.

[26] V. D. Phuc, V.-T. Tran, and N. V. Quyen. A novel design of the dynamic vibration absorbers for damped main systems under torsional excitation using least squares estimation of the equivalent linearization method. Journal of Multi-body Dynamics, 233, (1), (2019), pp. 60-70. https://doi.org/10.1177/1464419318779339.

[27] N. D. Anh and N. X. Nguyen. Extension of equivalent linearization method to design of TMD for linear damped systems. Structural Control and Health Monitoring, 19, (6), (2012), pp. 565573. https://doi.org/10.1002/stc.446.

[28] A. Ghosh and B. Basu. A closed-form optimal tuning criterion for TMD in damped structures. Structural Control and Health Monitoring, 14, (4), (2007), pp. 681-692. https://doi.org/10.1002/stc.176. 